Magnetohydrodynamic damping of oscillations in low-Prandtl-number convection

Hof, B. and Juel, Anne and Mullin, T.

2005

MIMS EPrint: 2006.352

Manchester Institute for Mathematical Sciences

School of Mathematics

The University of Manchester

\footnotetext{
Reports available from: http://eprints.maths.manchester.ac.uk/

And by contacting: The MIMS Secretary

School of Mathematics

The University of Manchester

Manchester, M13 9PL, UK
} 


\title{
Magnetohydrodynamic damping of oscillations in low-Prandtl-number convection
}

\author{
By B. $\mathrm{HOF}^{1}$, A. JUEL ${ }^{2}$ AND T. MULLIN ${ }^{1}$ \\ ${ }^{1}$ School of Physics and Astronomy, The University of Manchester, \\ Manchester M13 9PL, UK \\ ${ }^{2}$ School of Mathematics, The University of Manchester, \\ Oxford Road, Manchester M13 9EP, UK \\ bjorn@ reynolds.ph.man.ac.uk
}

(Received 13 April 2005 and in revised form 1 August 2005)

We present the results of an experimental investigation of the effect of a magnetic field on the stability of convection in a liquid metal. A rectangular container of gallium is subjected to a horizontal temperature gradient and a uniform magnetic field is applied separately in three directions. The magnetic field suppresses the oscillation most effectively when it is applied in the vertical direction and is least efficient when applied in the direction of the temperature gradient. The critical temperature difference required for the onset of oscillations is found to scale exponentially with the magnitude of the magnetic field for all three orientations. Comparisons are made with available theory and qualitative differences are discussed.

\section{Introduction}

Convective flows driven by horizontal temperature differences commonly occur in heat and mass transfer problems such as geophysical flows and various engineering applications. The stability of such convective flows is of particular interest in crystal growth where convection arises because of the temperature gradient between the melt and the solidified crystal. In most practical applications these flows are timedependent and the resulting temperature fluctuations at the solidification front have been linked to the occurrence of layers with differing dopant concentrations, so-called striations, in the grown crystal (Müller \& Wiehelm 1964). Independently Hurle (1966) and Utech \& Flemmings (1966) demonstrated that striations can be eliminated if crystals are grown in a sufficiently strong magnetic field. Applying a magnetic field to the moving conducting fluid induces electrical currents which in turn interact with the magnetic field resulting in a damping of the flow. In modern crystal growth facilities magnetic fields are often applied to increase the homogeneity of crystals. The present study is concerned with a laboratory model of the horizontal Bridgman method which is used for the growth of crystals of a specific form (Müller \& Ostrogorsky 1993). In an industrial setting, the crucible containing the molten crystal is withdrawn horizontally from a furnace, resulting in a horizontal temperature difference which in turn drives convective flows in the melt. This configuration is also of considerable academic interest because of its relative simplicity which allows progress to be made using analytical and numerical models of the flows. 


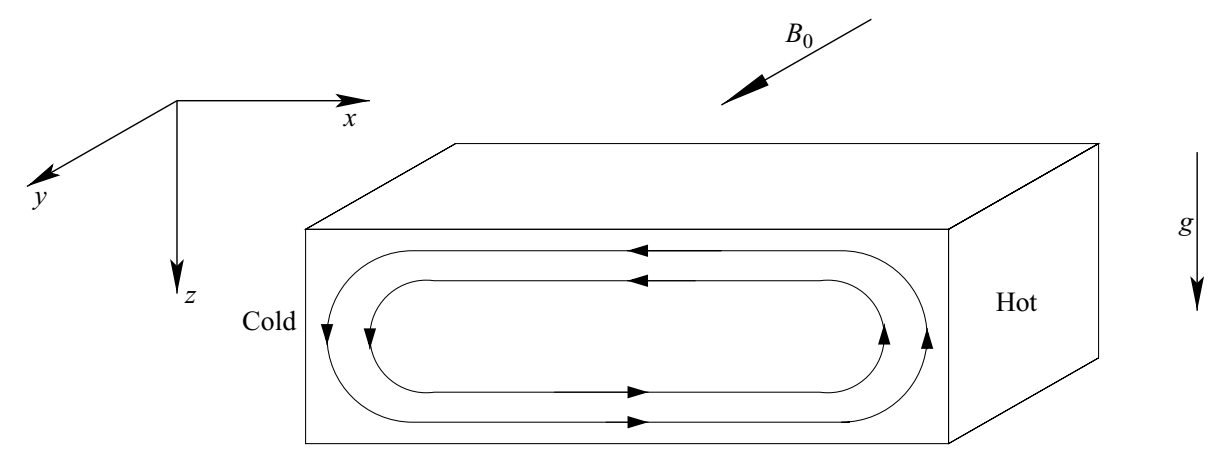

FIGURE 1 . Geometry of the experiment shown for a magnetic field $B_{0}$ of transverse orientation.

In our experiment we applied a controlled horizontal temperature difference to a sample of liquid gallium contained in an electrically and thermally insulating enclosure of rectangular cross-section. A uniform magnetic field $\left(B_{0}\right)$ was applied separately in the three principal orientations, namely the longitudinal (the magnetic field was parallel to the temperature gradient), the vertical (the field was parallel to gravity), and the transverse orientation (the magnetic field was perpendicular to both the temperature gradient and gravity). A schematic diagram of the geometry for the case of a magnetic field of transverse orientation is shown in figure 1 . The dimensionless parameters relevant to this problem are the Grashof, Hartmann and Prandtl numbers. The Grashof number is proportional to the applied temperature difference and it is a measure of the relative importance of buoyancy to viscous forces. It is defined as, $G r=\left(\alpha \Theta g h^{4}\right) /\left(l v^{2}\right)$ where $h(l)$ is the height (length) of the cavity, $v$ is the kinematic viscosity, $g$ the acceleration due to gravity, $\alpha$ the coefficient of thermal expansivity and $\Theta$ the applied temperature difference. The Hartmann number is defined as $H a=B_{0} h(\sigma / v \rho)^{1 / 2}$ where $\rho$ is the density, $B_{0}$ the magnetic field and $\sigma$ the electrical conductivity. $\mathrm{Ha}$ is proportional to the strength of the magnetic field and its square is a measure of the relative importance of the electromagnetic to the viscous forces. Finally the Prandtl number is defined as $P r=v / \kappa$ which is the ratio of the kinematic viscosity to the thermal diffusivity.

The pioneering experimental study of this problem was carried out by Hurle, Jakeman \& Johnson (1974) who investigated the dependence of the onset of oscillatory flows in liquid gallium on the strength of a transverse magnetic field. The gallium sample was held in a ceramic channel with a free upper surface and was subject to a horizontal temperature difference. The authors observed that the critical temperature difference for the onset of oscillations increases in proportion to the square of the field strength over the Hartmann number range investigated $(0<H a<17)$. In a later experimental study McKell et al. (1990) investigated the transition to chaos in the same apparatus and uncovered rich dynamical behaviour including a torusdoubling route to chaos. The effect of a magnetic field on the heat transfer in strongly time-dependent flows in a vertical slot has been addressed by Burr et al. (2003).

Kaddeche, Henry \& BenHadid (2003) investigated the stability of an infinite fluid layer confined by rigid top and bottom boundaries subject to a horizontal temperature difference and a magnetic field of vertical, transverse or longitudinal orientation. The vertical magnetic field stabilizes the flow most efficiently and suppresses transverse two-dimensional modes as well as longitudinal three-dimensional modes. For $\mathrm{Ha}<$ 7 the authors observed that $G r_{c} / G r_{c}(H a=0) \sim \exp \left(H a^{2}\right)$ for two-dimensional 
transverse modes and that $G r_{c} / G r_{c}(H a=0)-1 \sim H a^{2}$ for three-dimensional longitudinal modes, where $G r_{c}$ is the critical value of the Grashof number at which the instability occurs. Increasing $\mathrm{Ha}$ beyond approximately 15 leads to the disappearance of both modes of instability. The strong stabilization of the two-dimensional modes is directly connected to a strong reduction in the shear energy. The horizontal magnetic fields have far less impact. The transverse field only affects three-dimensional modes, whereas the longitudinal field only acts on two-dimensional modes. The stabilization is very weak at small $\mathrm{Ha}$ with an asymptotic behaviour at large $\mathrm{Ha}$ of $\mathrm{Gr}_{c} \sim \mathrm{Ha}$. In a similar study BenHadid, Henry \& Kaddeche (1997) investigated the stability of an infinite fluid layer with a free top surface in the presence of a vertical magnetic field. As in the case of a rigid surface, in the vertical field $G r_{c}$ for the transverse two-dimensional instability increases in proportion to $\exp \left(\mathrm{Ha}^{2}\right)$ whereas for the longitudinal three-dimensional mode $G r_{c}$ increases linearly with the square of the Hartmann number. Alboussiere, Henry \& Kaddeche (2003) focused on the limit of weak magnetic fields and showed analytically that in the limit of small $\mathrm{Ha}$ the Hopf bifurcation point increases in proportion to the square of the Hartmann number. By means of a linear stability analysis of the flow in an infinitely long cavity with a square cross-section, Bojarevics (1995) shows that the presence of lateral walls postpones the onset of oscillatory rolls in a vertical magnetic field.

Gelfgat \& Bar-Yoseph (2001) studied the stability of the flow in a laterally heated two-dimensional channel computationally using a Galerkin method. The aspect ratio (length/height) of the cell was 4 . Stability curves were calculated for various field orientations and the most effective suppression of oscillations was found for the vertical orientation, whereas the longitudinal field was the least efficient. The stability curves have a non-monotonic dependence on the Hartmann number, giving rise to hysteresis phenomena. Various different oscillatory modes are encountered at onset depending on the parameter settings.

In a recent combined experimental and numerical study of a gallium-filled cavity of aspect ratios 5.0:1.3:1.0 Hof et al. (2004) investigated the nature of the oscillatory instability. It was shown that oscillations set in at a supercritical Hopf bifurcation (see also Hof \& Mullin 2001). Excellent overall agreement was found between the experimental data and the direct numerical simulations for the spatial nature of the oscillation. The oscillatory mode was identified as a transverse standing wave where oscillation amplitudes measured close to the left sidewall display a $180^{\circ}$ phase shift compared to measurements taken close to the right sidewall. This oscillation is clearly of three-dimensional nature and therefore different from the instabilities encountered in the two-dimensional models reported above. These observations are in agreement with earlier experimental and numerical studies (Juel et al. 2001; Henry \& Buffat 1998).

The goal of this investigation is to establish the orientation of the magnetic field which damps the oscillations in the convective flow most efficiently. Experimental studies of this problem are of particular importance since the steady base flow (see Juel et al . 1999 for details) as well as the oscillatory mode are three-dimensional in nature. This questions the applicability of scaling laws obtained from two-dimensional models to practical flows.

\section{Experimental apparatus}

A detailed description of the experimental apparatus can be found in Hof, Juel \& Mullin (2003). Here we summarize the main features of the experiment. The sample 
of liquid gallium was held in an insulating enclosure of rectangular cross-section, which was machined from Lexan, a transparent plastic with a thermal conductivity 150 times smaller than that of gallium $\left(k=0.2 \mathrm{~W} \mathrm{~m}^{-1} \mathrm{~K}^{-1}\right)$. The conducting endwalls were made of $1 \mathrm{~mm}$ thick molybdenum sheets which have a thermal conductivity of approximately 5 times that of gallium. Molybdenum is also impervious to attack by gallium. Great care was taken to ensure that the cell was completely filled with gallium and sealed from the external environment. This ensured that all surfaces were wetted and oxidation of the gallium sample was avoided. Each molybdenum wall was inset into the side of a copper box through which silicone oil was circulated. The oil temperature was controlled to within $\pm 0.01{ }^{\circ} \mathrm{C}$ using a commercial circulator bath. In order to avoid heat losses of the gallium to the surrounding the cavity was thermally shielded as described in Hof et al. (2003).

The cavity was inserted between the iron cores of an electromagnet. It could be placed with its endwalls either parallel or perpendicular to the faces of the pole pieces, resulting in a longitudinal or transverse orientation of the magnetic fields respectively. A vertical field was applied by rotating the magnet through $90^{\circ}$. The magnetic fields were measured to be uniform to within $1.5 \%$ along the length of the enclosure and $0.5 \%$ across its width. Measurements of the convective flow were made using 14 type-K thermocouple probes of diameter $0.25 \mathrm{~mm}$ inserted by $1 \mathrm{~mm}$ into the gallium. The exact positions of the thermocouples are described in Hof et al. (2004). All thermocouples were carefully calibrated prior to the measurement, which enabled us to measure temperatures to an accuracy of $0.01 \mathrm{~K}$.

The experimental parameters $\mathrm{Gr}, \mathrm{Pr}$ and $\mathrm{Ha}$ are functions of the fluid properties and these vary with the temperature of the gallium sample. The temperature dependences of the material properties of gallium were discussed by Braunsfurth et al. (1997) and Hof et al. (2004). The values of the fluid parameters stated throughout this paper were calculated for the mean temperature in the channel, which was set to a constant value of $T_{\mathrm{m}}=75^{\circ} \mathrm{C}$. Thus the Prandtl number remained constant at $\operatorname{Pr}=0.018$. The range of attainable Grashof numbers was limited by the freezing point of gallium and the melting point of the Lexan channel. At a mean temperature of $T_{\mathrm{m}}=75^{\circ} \mathrm{C}$ the maximum value of the Grashof number that could be reached was $1.35 \times 10^{5}$. The experiment was fully automated and the sampling of the data, the temperature settings of the circulators and the regulation of the magnetic field were performed under computer control.

The investigation was focused on the onset of oscillations as a function of $G r$ and $H a$. The experimental protocol adopted was to first set $H a$ to a prescribed value where the flow was steady and then to increment the temperature difference between the hot and the cold wall in steps of $0.1 \mathrm{~K}(\Delta G r=300)$. Between increments the flow was left to settle for 30 minutes and temperatures were sampled for a further 30 minutes at a frequency of $5 \mathrm{~Hz}$. This process was repeated until the steady flow became unstable and oscillations set in. As described by Hof \& Mullin (2001) and Hof et al. (2004) the oscillatory flow arises at a supercritical Hopf bifurcation for variations of Grashof as well as Hartman numbers and no hysteretic dependence of the critical point was observed throughout the parameter regime investigated here. In order to detect the bifurcation point a fast Fourier transform (FFT) was automatically performed on each dataset. If the maximum of the FFT was at least one order of magnitude larger than the background noise a critical Grashof number was deemed to have been passed. From this weakly supercritical flow $\mathrm{Ha}$ was then incremented to a value at which the oscillation was suppressed and the flow was steady. The above procedure was repeated to determine the new bifurcation point and so forth. This 


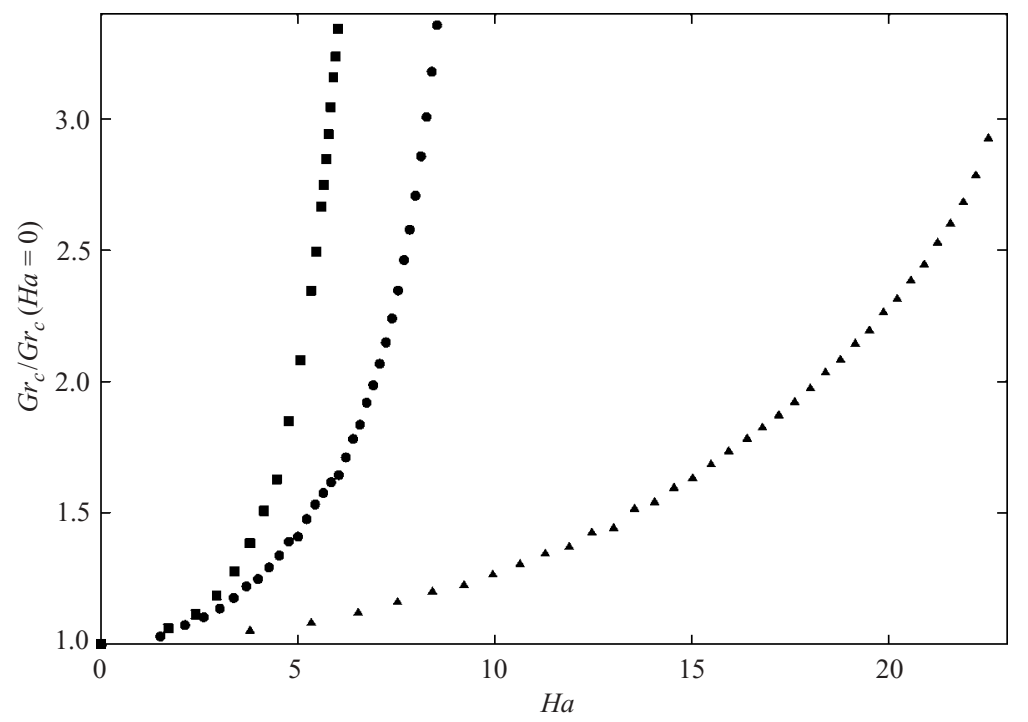

FIGURE 2. Stability curves: the data points mark the Hopf bifurcation points in the $(\mathrm{Gr}, \mathrm{Ha})$ plane. $\boldsymbol{\square}$, vertical; $\boldsymbol{\bullet}$, transverse; $\boldsymbol{\Delta}$, longitudinal magnetic field.

automation process, and in particular the long settling times between increments, enabled the accurate determination of the onset of oscillations. The error in the critical Grashof number was less than one step size, which is typically $\Delta G r=300$ and less than $1 \%$ of the absolute value.

\section{Hartmann number dependence of the oscillation}

One set of experiments was performed, for each of the principal directions of the magnetic field (transverse, longitudinal and vertical). Changing the orientation of the magnetic field between the three sequences of experiments involved major changes to the experimental set up, including emptying and refilling of the gallium channel. This resulted in a displacement of the critical Grashof number $G r_{c}$ at $H a=0$, which is small in comparison to the range of Grashof numbers investigated. The critical points found in the three experiments at zero Hartmann number are $G r_{c}(H a=0)=3.91 \times 10^{4}$, for the transverse field, $G r_{c}(H a=0)=4.15 \times 10^{4}$ for the longitudinal field and $\mathrm{Gr}_{c}(\mathrm{Ha}=0)=4.03 \times 10^{4}$ for the vertical field.

The stability curves of the three orientations of the magnetic field are shown in figure 2. For parameter settings below (above) the curves, flows were steady (oscillatory). To enable a clear comparison between the three measurements, the Grashof number has been rescaled in terms of its critical value at $H a=0$. Magnetic fields applied in each of the three orientations suppress oscillations and the Hopf bifurcation point for the onset of oscillations is shifted to larger Grashof numbers with increasing Hartmann numbers. The same mode of oscillation was observed throughout the investigated parameter regime. The vertical field is the most efficient at suppressing oscillations, and the longitudinal field shows a significantly weaker effect than either the vertical or the transverse orientation. $\mathrm{Ha}=5.5$ and $\mathrm{Ha}=8$ in the vertical and the transverse field respectively are sufficient to stabilize the steady flow up to $G r=1.1 \times 10^{5}\left(G r_{c} / G r_{c}(H a=0)=2.75\right)$. However for the longitudinal field a Hartmann number of 22 is required to move the bifurcation 


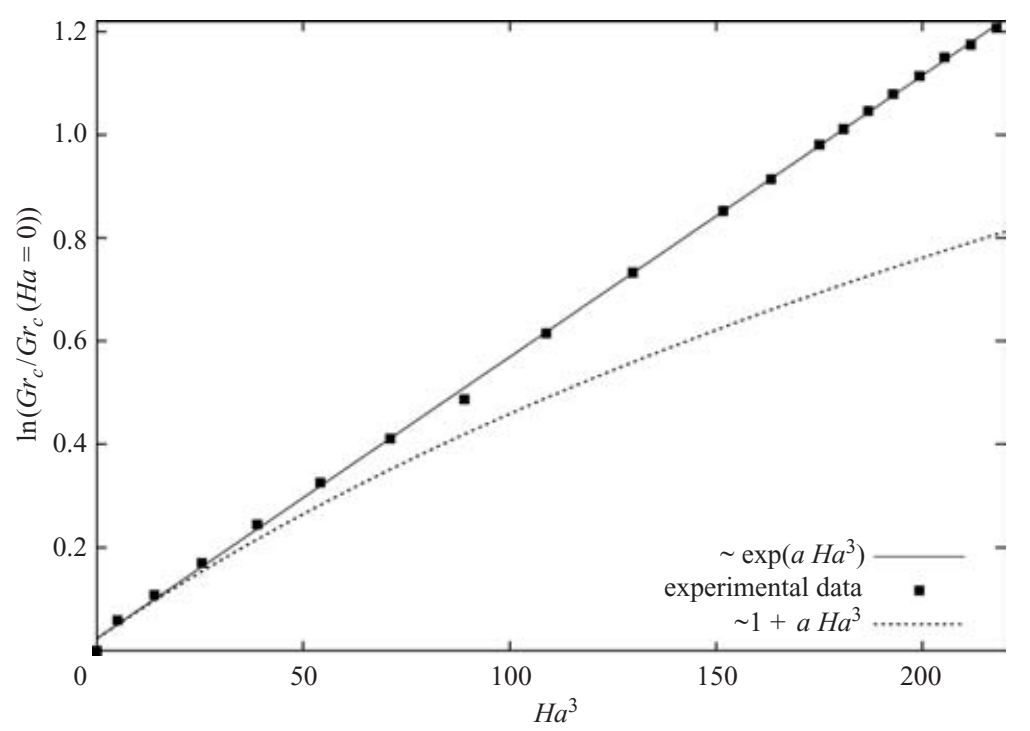

Figure 3. The natural logarithm of the rescaled critical Grashof number is plotted as a function of $\mathrm{Ha}^{3}$ for the vertical field. The fitted (solid) line indicates that the critical value of $G r_{c} / G r_{c}(H a=0)$ for the onset of oscillations scales in proportion to $\exp \left(a H a^{3}\right)$, where $a=0.0055$. The dotted curve corresponds to the first two terms of the Taylor expansion of the fitted exponential.

point by the same amount. Hence the vertical and transverse fields damp oscillations more efficiently than the longitudinal magnetic field. In two-dimensional numerical calculations Gelfgat \& Bar-Yoseph (2001) also observe a much stronger damping for the vertical than for the longitudinal field. Interestingly for the damping effect on steady flows, where considerably larger field strengths are required, Hof et al. (2003) observe that the transverse field is only marginally more effective than the longitudinal field. Both were found to be far less efficient than the vertical magnetic field, whereas here for the suppression of oscillations both the vertical and the transverse field are very effective.

The dependence of the critical value of $\mathrm{Gr}$ on $\mathrm{Ha}$ is given in figures 3, 4 and 5 for the vertical, transverse and longitudinal fields respectively. Here $\ln \left(G r_{c} / G r_{c}(H a=0)\right)$ is plotted against a suitable power of $H a$. In the case of the vertical field (figure 3), $G r_{c} \propto \exp \left((0.00546 \pm 0.00003) H a^{3}\right)$ and this functional dependence is accurate over the entire range of $\mathrm{Ha}$ investigated. The dotted curve shown in the figure corresponds to the first two terms of a Taylor expansion of the exponential fit. The curve begins to deviate from the data points for $H a>3$. This clearly shows that the data follow an exponential scaling, in contrast with the power-law behaviour suggested by the stability analysis of Kaddeche et al. (2003). Plotting $\ln G r_{c} / G r_{c}(H a=0)$ versus $\mathrm{Ha}^{2}$ for the transverse field, as shown in figure 4, suggests that the critical point increases as $\exp \left((0.0141 \pm 0.0001) H a^{2}\right)$. However, for $H a>7$, the scaling changes and $G r_{c}$ increases more strongly. Changes in the scaling of the stability curves were also observed by Gelfgat \& Bar-Yoseph (2001) which occurred at parameter settings where two modes intersect. In the experiment the mode of the oscillation remained unchanged over the parameter range investigated. Again the power expansion of the exponential fit, shown by the dotted curve, only holds for $\mathrm{Ha}<3$.

The scaling of $G r_{c}$ for the longitudinal field is found to be $G r_{c} \propto \exp \left(H a^{2}(0.00201 \pm\right.$ $0.00001)$ ) as shown in figure 5. A quadratic scaling with Hartmann number is only 


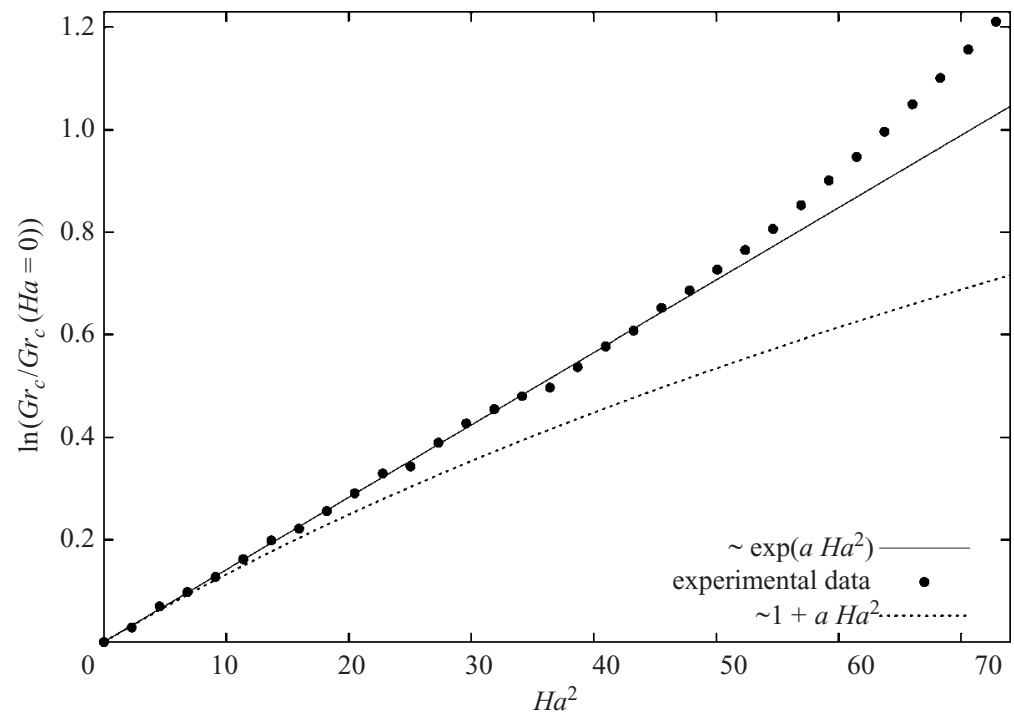

FIGURE 4. The natural logarithm of the rescaled critical Grashof number is plotted versus $\mathrm{Ha}^{2}$ for the transverse field. The fitted line indicates that the Hopf bifurcation point scales in proportion to $\exp \left(a H a^{2}\right)$ for $H a \lesssim 7$, with $a=0.0141$. The dotted curve corresponds to the first two terms of the Taylor expansion of the fitted exponential.

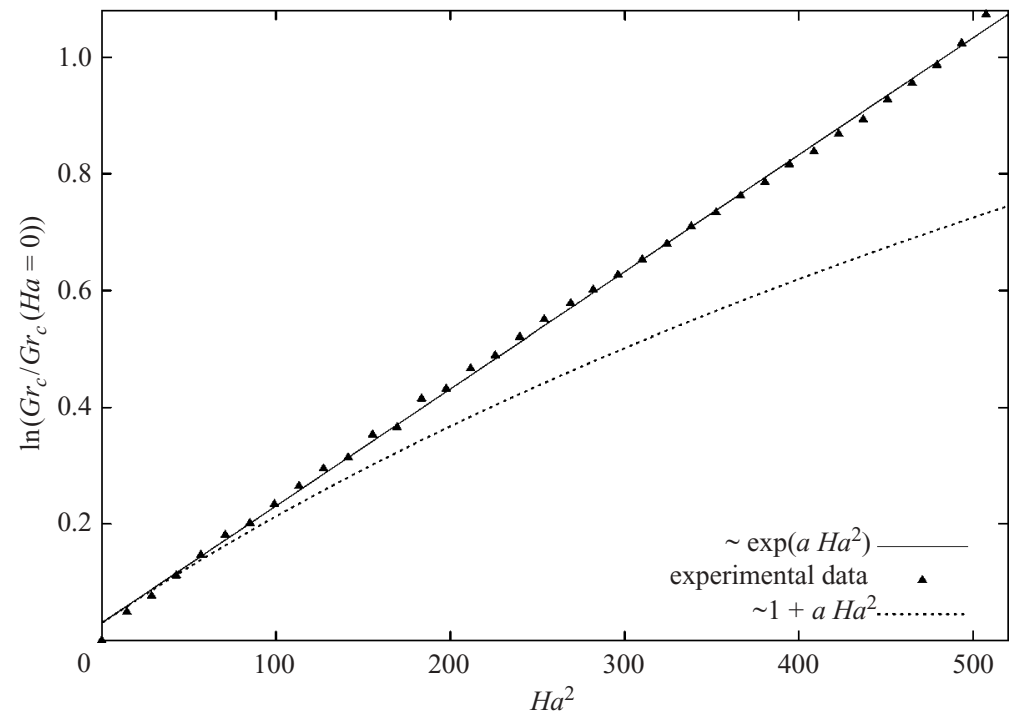

FIGURE 5. The natural logarithm of the rescaled critical Grashof number is plotted versus $\mathrm{Ha}^{2}$ for the longitudinal field. The linear fit indicates that the Hopf bifurcation point scales in proportion to $\exp \left(a H a^{2}\right)$, with $a=0.002$. The dotted curve corresponds to the first two terms of the Taylor expansion of the fitted exponential.

observed for $\mathrm{Ha}<10$ (see dotted curve in figure 5). The scalings observed in this study clearly do not agree with those of the two-dimensional analytical models discussed in $\$ 2$. One reason for the different scaling behaviour found is that the instability in the model is qualitatively different from that in the experiment. Specifically a three-dimensional standing wave is observed in the experiment whereas longitudinal 


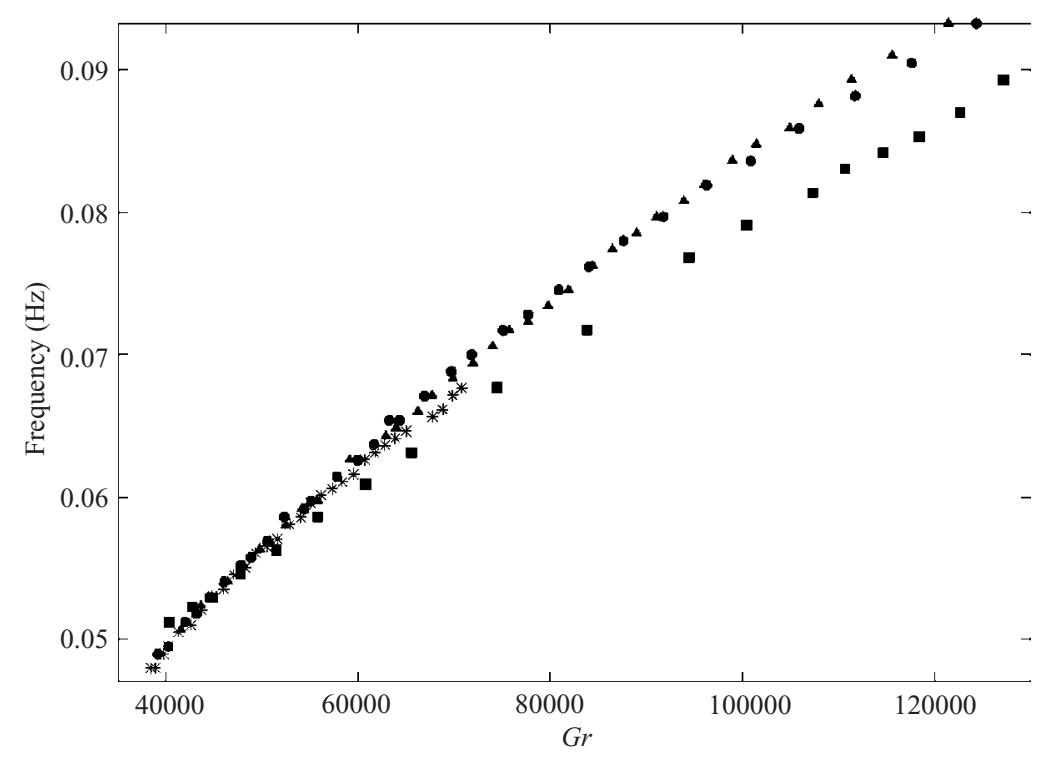

FIGURE 6. Scaling of the frequency with Grashof number: $\mathbf{\square}$, vertical magnetic field; transverse magnetic field; $\boldsymbol{\Delta}$, longitudinal magnetic field; *, no magnetic field.

oscillatory rolls or transverse rolls are encountered in the infinite-layer model. However it is interesting to note that an $\exp \left(H a^{2}\right)$ scaling for $G r_{c}$ which we observed in the longitudinal and transverse field has been observed by Kaddeche et al. (2003) and BenHadid et al. (1997) for the damping of the two-dimensional transverse instability by a vertical magnetic field. It is also evident that the scaling observed for the transverse field is different from the $G r_{c} \propto H a^{2}$ scaling found by Hurle et al. (1974) for free-surface flows. Whereas Hurle et al. (1974) observed a quadratic scaling up to $H a=17$ our data only show a quadratic behaviour up to $H a=3$.

In figure 6 the frequencies measured at each of the critical points are shown plotted against the Grashof number. For the transverse and longitudinal magnetic fields the frequency shows the same dependence on $\mathrm{Gr}$ and this also agrees with the frequency dependence found in the absence of a magnetic field. Hence, the frequency of the oscillation is largely independent of $\mathrm{Ha}$ and only depends on the Grashof number. For the vertical field, however, the frequency is affected by the magnetic field and the increase of the frequency is less steep than in the other cases. As shown by Hof et al. (2003) the temperature field of the steady flow is considerably affected for $H a \approx 5$ in a vertical magnetic field, whereas in the transverse and longitudinal fields Hartmann numbers an order of magnitude larger are required to have the same effect on the temperature field. The different frequency scaling found for the vertical field could therefore be caused by modifications of the base flow by the vertical magnetic field.

\section{Conclusion}

We have shown that the onset of oscillations occurring in low-Prandtl-number convection can be postponed using magnetic fields of relatively small magnitude. The size of the field required is approximately one order of magnitude smaller than that necessary to significantly damp the steady flow (Hof et al. 2003). The efficiency of the magnetic field strongly depends on its orientation with respect to the temperature gradient. Exponential scalings of the Hopf bifurcation point are 
observed over the investigated parameter range for all three field orientations. These results clearly differ from the two-dimensional analysis and underline the relevance of the three-dimensional structure of the flow. The most surprising observation is the strong suppression of oscillations by the transverse field. From the results of the stability analysis by Kaddeche et al. (2003) a substantially stronger damping was expected for the vertical field than for the transverse field. In the experiment however, both the vertical and the transverse magnetic fields show a strong suppression of oscillations compared with the longitudinal field.

The authors wish to thank Daniel Henry for helpful discussions. This work was supported by the EPSRC (B.H.), AEA Technology, Harwell (B.H.), an Alliance Partnership grant from the British Council (A.J.), an EPSRC advanced research fellowship (A.J.) and an EPSRC Senior Fellowship (T.M.).

\section{REFERENCES}

Alboussiere, T., Henry, D. \& Kaddeche, S. 2003 Note on braking and stabilization laws for buoyant flows under a weak magnetic field. Fluid Dyn. Res. 13, 287-297.

BenHadid, H., Henry, D. \& Kaddeche, S. 1997 Numerical study of convection in the horizontal bridgman configuration under the action of a constant magnetic field. Part 1. Two-dimensional flow. J. Fluid Mech. 333, 23-56.

BoJAREVICS, V. 1995 Buoyancy driven flow and its stability in a horizontal rectangular channel with an arbitrary oriented transversal magnetic field. Magnetohydrodynamics 31, 245-253.

Braunsfurth, M., Skeldon, A., Juel, A., Mullin, T. \& Riley, D. 1997 Free convection in liquid gallium. J. Fluid Mech. 342, 295-314.

Burr, U., Barleon, L., Jochmann, P. \& Tsinober, A. 2003 Magnetohydrodynamic convection in a vertical slot with horizontal magnetic field. J. Fluid Mech. 475, 21-40.

Gelfgat, A. \& Bar-Yoseph, P. 2001 The effect of an external magnetic field on oscillatory instability of convective flows in a rectangular cavity. Phys. Fluids 13, 2269-2278.

Henry, D. \& Buffat, M. 1998 Two and three-dimensional numerical simulations of the transition to oscillatory convection in low-prandtl number fluids. J. Fluid Mech. 374, 145-171.

Hof, B., JuEl, A. \& Mullin, T. 2003 Magnetohydrodynamic damping of convective flows in molten gallium. J. Fluid Mech. 482, 163-179.

Hof, B., Juel, A., Zhao, L., BenHadid, H., Henry, D. \& Mullin, T. 2004 On the onset of oscillatory convection in molten gallium. J. Fluid Mech. 515, 391-413.

Hof, B. \& Mullin, T. 2001 Critical dynamics in oscillatory convection. Magnetohydrodynamics 37, $119-126$.

HurLe, D. 1966 Temperature oscillations in molten metals and their relationship to growth striae in melt-grown crystals. Phil. Mag. 13, 305-310.

Hurle, D., Jakeman, E. \& Johnson, C. 1974 Convective temperature oscillations in molten gallium. J. Fluid Mech. 64, 565-576.

Juel, A., Mullin, T., BenHadid, H. \& Henry, D. 1999 Magnetohydrodynamic convection in molten gallium. J. Fluid Mech. 378, 97-118.

Juel, A., Mullin, T., BenHadid, H. \& Henry, D. 2001 Three-dimensional free convection in molten gallium. J. Fluid Mech. 436, 267-281.

Kaddeche, S., Henry, D. \& BenHadid, H. 2003 Magnetic stabilization of the buoyant convection between infinite horizontal walls with a horizontal temperature gradient. J. Fluid Mech. 480, $185-216$.

McKell, K., Broomhead, D., Jones, R. \& Hurle, D. 1990 Torus doubling in convecting molten gallium. Europhys. Lett. 12, 513-518.

MülleR, A. \& WieHELM, M. 1964 Periodische temperaturschwankungen in fluessigem insb als ursache schichtweisen einbaus von te in kristallisierendes insb. Z. Naturf. A 19, 254-263.

Müller, G. \& Ostrogorsky, A. 1993 Convection in melt growth. In Handbook of Crystal Growth: Growth Mechanisms and Dynamics (ed. D. T. J. Hurle), vol. 2b. North-Holland.

Utech, H. \& Flemings, M. 1966 Elimination of solute banding in indium antimonide crystals by growth in a magnetic field. J. App. Phys. 37, 2021-2024. 